\title{
Determining constant breadth curve mate of a curve on a surface via Taylor collocation method
}

\author{
Burak Sahiner and Mehmet Sezer \\ Department of Mathematics, Manisa Celal Bayar University, Manisa, Turkey \\ Received: 12 February 2018, Accepted: 10 June 2018 \\ Published online: 7 August 2018.
}

\begin{abstract}
In this paper, a system of differential equations characterizing constant breadth curve mate of a curve on a surface is given. It is shown that a collocation method based on Taylor polynomials can be used to find approximate solutions of the system. Then, an example is given to illustrate the efficiency of the method.
\end{abstract}

Keywords: Curves of constant breadth, Darboux frame, Taylor collocation method, System of differential equations

\section{Introduction}

Curves of constant breadth which were introduced by L. Euler [6] in 1778 have attracted many authors' attention since that date. Especially, after Reuleaux [17] has found applications of curves of constant breadth in kinematics of machinery, particularly, in cam-follower mechanisms, interest of this subject has rapidly increased.

Initial works in the subject of curves of constant breadth were proposed in plane by Euler [6], Barbier [2], and Mellish [15]. Later, this subject was studied in many different spaces by many authors, for example, in Euclidean 3-space by Fujiwara [7], in Euclidean 4-space by Mağden and Köse [14], in Euclidean n-space by Akdoğan and Mağden [1], in Minkowski 3-space by Kocayiğit and Önder [11] and Yılmaz and Turgut [20], in Minkowski 4-space by Kazaz et al. [8]. Furthermore, many authors contributed to the research area of curves of constant breadth. Blaschke [3] defined curves of constant breadth on sphere, Köse $[12,13]$ presented some concepts of space curves of constant breadth and gave some geometric properties for curves of constant breadth in plane, Kocayiğit and Çetin [9] studied space curves of constant breadth according to Bishop frame.

One of the important problems in the research area of constant breadth curves is obtaining differential equations or system of differential equations characterizing curves of constant breadth. This problem was handled by Sezer in Euclidean 3-space [18]. Later, Önder et al. [16] and Kocayiğit and Çiçek [10] also obtained differential equations characterizing curves of constant breadth in Minkowski 3-space and Minkowski 4-space, respectively. These differential equations have variable coefficients, so one cannot find analytical solutions of them easily. In the works of [4] and [5], approximate solutions of differential equations characterizing space curves of constant breadth were obtained by using collocation methods based on Lucas polynomials and Taylor polynomials, respectively.

In the present paper, we try to determine a curve which is a constant breadth curve mate of a curve on a surface. To accomplish this, we first give a system of differential equations characterizing constant breadth curve mate according to Darboux frame. This system of differential equations has variable coefficients. So it is hard to find analytical solutions of 
the system. By using Taylor collocation method, we find approximate solutions of the system. Thus, we determine the constant breadth curve mate approximately. Finally, by giving an example, we illustrate that this technique is efficient to determine the constant breadth curve mate.

\section{A brief overview of Darboux frame of a curve on a surface}

In this section, we briefly introduce Darboux frame and its derivation formulas of a curve on a surface.

Let a surface $M$ be given as $X(u, v)$ and $\alpha$ be a unit speed curve on the surface $M$. The unit tangent vector field of the curve $\alpha$ and the unit normal vector field of the surface $M$ along the curve $\alpha$ can be given, respectively, as

$$
T=\alpha^{\prime}
$$

and

$$
n_{\alpha}=\frac{X_{u} \times X_{v}}{\left\|X_{u} \times X_{v}\right\|}(\alpha)
$$

where $X_{u}$ and $X_{v}$ denote the partial derivatives of $X(u, v)$ with respect to $u$ and $v$, respectively. If we define a unit vector $g=n_{\alpha} \times T$, we will have a frame which is called Darboux frame. The Darboux frame is the analog of the Serret-Frenet frame of a curve and is used to study differential geometry of a curve embedded in a surface. The derivative formulae of the Darboux frame can be given in matrix form as

$$
\left[\begin{array}{c}
T^{\prime} \\
g^{\prime} \\
n_{\alpha}^{\prime}
\end{array}\right]=\left[\begin{array}{ccc}
0 & k_{g} & k_{n} \\
-k_{g} & 0 & \tau_{g} \\
-k_{n} & -\tau_{g} & 0
\end{array}\right]\left[\begin{array}{c}
T \\
g \\
n_{\alpha}
\end{array}\right]
$$

where $k_{g}, k_{n}$, and $\tau_{g}$ are called geodesic curvature, normal curvature, and geodesic torsion, respectively.

\section{System of differential equations of constant breadth curve pair}

In this section, we obtain a system of differential equations characterizing a constant breadth curve mate of a curve on a surface by the aid of Darboux frame mentioned in Section 2. First, we will give the following definition of a curve pair of constant breadth.

Definition 1. [12] A pair of curves $\alpha$ and $\alpha^{*}$ in $E^{3}$, for which the tangents at the corresponding points $\alpha(s)$ and $\alpha^{*}\left(s^{*}\right)$ are parallel and in opposite directions, and the distance between these points is always constant, is called constant breadth curve pair.

Let $\alpha$ be a unit speed curve on a surface $M$. If $\alpha^{*}$ is a constant breadth curve pair of $\alpha$, then we can write

$$
\alpha^{*}\left(s^{*}\right)=\alpha(s)+\lambda_{1}(s) T(s)+\lambda_{2}(s) g(s)+\lambda_{3}(s) n_{\alpha}(s)
$$

where $T, g$ and $n_{\alpha}$ are elements of Darboux frame of $\alpha$, and $\lambda_{i},(i=1,2,3)$ are functions of $s$. By differentiating equation (2) and using equation (1), we have

$$
\frac{d \alpha^{*}}{d s}=\frac{d \alpha^{*}}{d s^{*}} \frac{d s^{*}}{d s}=\left(1+\lambda_{1}^{\prime}-\lambda_{2} k_{g}-\lambda_{3} k_{n}\right) T+\left(\lambda_{1} k_{g}+\lambda_{2}^{\prime}-\lambda_{3} \tau_{g}\right) g+\left(\lambda_{1} k_{n}+\lambda_{2} \tau_{g}+\lambda_{3}^{\prime}\right) n,
$$

where $s^{*}$ is the arc-length parameter of $\alpha^{*}$, and $k_{g}, k_{n}$, and $\tau_{g}$ are geodesic curvature, normal curvature, and geodesic torsion of the curve $\alpha$, respectively. $\left\langle T, T^{*}\right\rangle=-1$ is the necessary and sufficient condition for $\alpha^{*}$ be a constant breadth 
curve mate of $\alpha$, where $T$ and $T^{*}$ are unit tangent vector fields of the curves $\alpha$ and $\alpha^{*}$, respectively. Thus, from equation (3), we obtain the following system of differential equations

$$
\left.\begin{array}{l}
\lambda_{1}^{\prime}=k_{g} \lambda_{2}+k_{n} \lambda_{3}-\frac{d s^{*}}{d s}-1 \\
\lambda_{2}^{\prime}=-k_{g} \lambda_{1}+\tau_{g} \lambda_{3} \\
\lambda_{3}^{\prime}=-k_{n} \lambda_{1}-\tau_{g} \lambda_{2}
\end{array}\right\} .
$$

On the other hand, from equation (2), we can write

$$
\alpha^{*}-\alpha=\lambda_{1} T+\lambda_{2} g+\lambda_{3} n_{\alpha}
$$

Since $\alpha$ and $\alpha^{*}$ are the constant breadth curve pair, the distance between these curves can be constant, i.e.,

$$
\left\|\alpha^{*}-\alpha\right\|=\sqrt{\lambda_{1}^{2}+\lambda_{2}^{2}+\lambda_{3}^{2}}=c
$$

where $c$ is a constant. By differentiating equation (5), we get

$$
\lambda_{1} \lambda_{1}^{\prime}+\lambda_{2} \lambda_{2}^{\prime}+\lambda_{3} \lambda_{3}^{\prime}=0
$$

By substituting system (4) into equation (6), we obtain the following equation which characterizes constant breadth curve pair $\alpha$ and $\alpha^{*}$ :

$$
\lambda_{1}\left(-\frac{d s^{*}}{d s}-1\right)=0
$$

From the above equation, two cases should be considered.

Case 1. In the first case, we assume that $-\frac{d s^{*}}{d s}-1=0$. Therefore, the system (4) becomes

$$
\left.\begin{array}{l}
\lambda_{1}^{\prime}=k_{g} \lambda_{2}+k_{n} \lambda_{3} \\
\lambda_{2}^{\prime}=-k_{g} \lambda_{1}+\tau_{g} \lambda_{3} \\
\lambda_{3}^{\prime}=-k_{n} \lambda_{1}-\tau_{g} \lambda_{2}
\end{array}\right\}
$$

The system (7) is a system of differential equations with variable coefficients in normal form. Given a curve $\alpha$ and the initial conditions $\lambda_{1}(0)=\mu_{1}, \lambda_{2}(0)=\mu_{2}, \lambda_{3}(0)=\mu_{3}$ which determine the initial point of $\alpha^{*}$, where $\mu_{1}, \mu_{2}$ and $\mu_{3}$ are real numbers. This system which gives the constant breadth curve mate $\alpha^{*}$ of $\alpha$ can be solved approximately by using Taylor collocation method to be described in Section 4.

Case 2. In the second case, we assume that $\lambda_{1}=0$. Then, the system (4) becomes

$$
\left.\begin{array}{l}
\lambda_{1}^{\prime}=k_{g} \lambda_{2}+k_{n} \lambda_{3}-\frac{d s^{*}}{d s}-1=0 \\
\lambda_{2}^{\prime}=\tau_{g} \lambda_{3} \\
\lambda_{3}^{\prime}=-\tau_{g} \lambda_{2}
\end{array}\right\}
$$


From the second and third equations of the system (8), we obtain

$$
\lambda_{2}=\sin \left(\int \tau_{g} d s\right)
$$

and

$$
\lambda_{3}=\cos \left(\int \tau_{g} d s\right) .
$$

By considering the initial conditions $\lambda_{2}(0)=\mu_{2}$ and $\lambda_{3}(0)=\mu_{3}$, where $\mu_{2}$ and $\mu_{3}$ are real numbers, $\lambda_{2}$ and $\lambda_{3}$ can be determined. By substituting $\lambda_{2}$ and $\lambda_{3}$ into the first equation of the system (8), the ratio $\frac{d s^{*}}{d s}$ can be found.

\section{Taylor collocation method for system of linear differential equations with variable coefficients in normal form}

In order to find approximate solutions of systems of high-order linear differential equations with variable coefficients, a Taylor collocation method was given in [19]. In [5], this method was adapted for the systems of three linear differential equations. In this study, we use the method described in [5] to obtain approximate solutions of the system of differential equations characterizing constant breadth curve mate of a curve on a surface. In this section, we briefly introduce Taylor collocation method for system of linear differential equations with variable coefficients in normal form (see [5] for details). The system of three linear differential equations with variable coefficients in the normal form

$$
L\left[y_{i}(x)\right]=y_{i}^{\prime}(x)-\sum_{j=1}^{3} p_{i, j}(x) y_{j}(x)=g_{i}(x), \quad(i=1,2,3), \quad(0 \leq a \leq x \leq b)
$$

under the initial conditions

$$
y_{i}(a)=c_{i}
$$

where $y_{i}(x)(i=1,2,3)$ are unknown functions, $p_{i, j}(x)$ and $g_{i}(x)$ are the known continuous functions defined on interval $[a, b]$, and $c_{i}(i=1,2,3)$ are real constants. By using the Taylor collocation method, the approximate solutions of the system (9) can be obtained as

$$
y_{i} \cong y_{i, N}(x)=\sum_{n=0}^{N} a_{i, n} x^{n}
$$

which is the Taylor polynomial solution, where $a_{i, n},(n=0,1,2, \ldots, N)$ is the unknown Taylor coefficients.

In order to find the solutions of the system (9) under the initial conditions (10), we can use the collocation points defined by

$$
x_{k}=a+\frac{b-a}{N} k, \quad k=0,1, \ldots, N, \quad 0 \leq a \leq x \leq b .
$$

On the other hand, we can write the approximate solutions $y_{i, N}(x)$ given by Eq.(11) in the matrix form

$$
y_{i, N}(x)=\mathbf{X}(x) \mathbf{A}_{i},(i=1,2,3)
$$

where

$$
\mathbf{X}(x)=\left[\begin{array}{lllll}
1 & x & x^{2} & \ldots & x^{N}
\end{array}\right]
$$

and

$$
\mathrm{A}_{i}=\left[\begin{array}{lllll}
a_{i, 0} & a_{i, 1} & a_{i, 2} & \ldots & a_{i, N}
\end{array}\right]^{T} .
$$


From Eq.(13), the solutions $y_{i, N}(x),(i=1,2,3)$ can be expressed as

$$
\mathrm{Y}(x)=\overline{\mathrm{X}}(x) \mathrm{A}
$$

where

$$
\mathbf{Y}(x)=\left[\begin{array}{l}
y_{1, N}(x) \\
y_{2, N}(x) \\
y_{3, N}(x)
\end{array}\right], \overline{\mathbf{X}}(x)=\left[\begin{array}{ccc}
\mathbf{X}(x) & 0 & 0 \\
0 & \mathbf{X}(x) & 0 \\
0 & 0 & \mathbf{X}(x)
\end{array}\right], \mathbf{A}=\left[\begin{array}{c}
\mathbf{A}_{1} \\
\mathbf{A}_{2} \\
\mathbf{A}_{3}
\end{array}\right] .
$$

Also, the relation between the matrix $\mathrm{X}(x)$ and its derivative $\mathrm{X}^{\prime}(x)$ is

$$
\mathbf{X}^{\prime}(x)=\mathbf{X}(x) \mathbf{B}
$$

where

$$
\mathbf{B}=\left[\begin{array}{cccccc}
0 & 1 & 0 & 0 & \ldots & 0 \\
0 & 0 & 2 & 0 & \cdots & 0 \\
0 & 0 & 0 & 3 & \ldots & 0 \\
\vdots & \vdots & \vdots & \vdots & \ddots & \vdots \\
0 & 0 & 0 & 0 & \cdots & N \\
0 & 0 & 0 & 0 & \cdots & 0
\end{array}\right] .
$$

By using the relations (13) and (15), we obtain the following matrix relation

$$
y_{i, N}^{\prime}(x)=\mathbf{X}(x) \mathbf{B} \mathbf{A}_{i}, \quad(i=1,2,3) .
$$

Therefore, we can write the following matrix relation

$$
\mathbf{Y}^{\prime}(x)=\overline{\mathbf{X}}(x) \overline{\mathbf{B}} \mathbf{A}
$$

where

$$
\mathbf{Y}^{\prime}(x)=\left[\begin{array}{l}
y_{1, N}^{\prime}(x) \\
y_{2, N}^{\prime}(x) \\
y_{3, N}^{\prime}(x)
\end{array}\right], \overline{\mathbf{B}}=\left[\begin{array}{lll}
\mathbf{B} & 0 & 0 \\
0 & \mathbf{B} & 0 \\
0 & 0 & \mathbf{B}
\end{array}\right] .
$$

On the other hand, the system (9) can be written in matrix form as

$$
\mathrm{Y}^{\prime}(x)=\mathrm{P}(x) \mathrm{Y}(x)+\mathrm{G}(x)
$$

where

$$
\mathrm{P}(x)=\left[\begin{array}{lll}
p_{1,1}(x) & p_{1,2}(x) & p_{1,3}(x) \\
p_{2,1}(x) & p_{2,2}(x) & p_{2,3}(x) \\
p_{3,1}(x) & p_{3,2}(x) & p_{3,3}(x)
\end{array}\right], \mathrm{G}(x)=\left[\begin{array}{l}
g_{1}(x) \\
g_{2}(x) \\
g_{3}(x)
\end{array}\right] .
$$

By using the collocation points given by (12) into Eq.(17), we obtain the system of matrix equations

$$
\mathrm{Y}^{\prime}\left(x_{k}\right)=\mathrm{P}\left(x_{k}\right) \mathrm{Y}\left(x_{k}\right)+\mathrm{G}\left(x_{k}\right), \quad(k=0,1, \ldots, N) .
$$

This matrix equation can also be written briefly as

$$
\mathrm{Y}^{\prime}=\mathrm{PY}+\mathrm{G}
$$


where

$$
\mathrm{P}=\left[\begin{array}{cccc}
\mathrm{P}\left(x_{0}\right) & 0 & \cdots & 0 \\
0 & \mathrm{P}\left(x_{1}\right) & \cdots & 0 \\
\vdots & \vdots & \ddots & \vdots \\
0 & 0 & \cdots & \mathrm{P}\left(x_{N}\right)
\end{array}\right], \mathrm{Y}=\left[\begin{array}{c}
\mathrm{Y}\left(x_{0}\right) \\
\mathrm{Y}\left(x_{1}\right) \\
\vdots \\
\mathrm{Y}\left(x_{N}\right)
\end{array}\right], \mathrm{Y}^{\prime}=\left[\begin{array}{c}
\mathrm{Y}^{\prime}\left(x_{0}\right) \\
\mathrm{Y}^{\prime}\left(x_{1}\right) \\
\vdots \\
\mathrm{Y}^{\prime}\left(x_{N}\right)
\end{array}\right], \mathrm{G}=\left[\begin{array}{c}
\mathrm{G}\left(x_{0}\right) \\
\mathrm{G}\left(x_{1}\right) \\
\vdots \\
\mathrm{G}\left(x_{N}\right)
\end{array}\right]
$$

From the relations (14) and (16) and the collocation points given by (12), we obtain

$$
\mathbf{Y}\left(x_{k}\right)=\overline{\mathbf{X}}\left(x_{k}\right) \mathbf{A} \text { and } \mathbf{Y}^{\prime}\left(x_{k}\right)=\overline{\mathbf{X}}\left(x_{k}\right) \overline{\mathbf{B}} \mathbf{A}, \quad(k=0,1, \ldots, N)
$$

or briefly

$$
\mathbf{Y}=\mathbf{X A} \text { and } \mathbf{Y}^{\prime}=\mathbf{X} \overline{\mathbf{B}} \mathbf{A}
$$

where

$$
\mathrm{X}=\left[\begin{array}{c}
\overline{\mathrm{X}}\left(x_{0}\right) \\
\overline{\mathrm{X}}\left(x_{1}\right) \\
\vdots \\
\overline{\mathrm{X}}\left(x_{N}\right)
\end{array}\right], \overline{\mathrm{X}}\left(x_{k}\right)=\left[\begin{array}{cccc}
\mathrm{X}\left(x_{k}\right) & 0 & \cdots & 0 \\
0 & \mathrm{X}\left(x_{k}\right) & \cdots & 0 \\
\vdots & \vdots & \ddots & \vdots \\
0 & 0 & \cdots & \mathrm{X}\left(x_{k}\right)
\end{array}\right]
$$

By substituting the relations given by (19) into Eq.(18), we obtain the matrix equation as

$$
\{\mathrm{X} \overline{\mathrm{B}}-\mathrm{PX}\} \mathrm{A}=\mathrm{G}
$$

In Eq.(20), the dimensions of the matrices $\mathrm{P}, \mathrm{X}, \overline{\mathrm{B}}, \mathrm{A}$ and $\mathrm{G}$ are $3(N+1) \times 3(N+1), 3(N+1) \times 3(N+1)$, $3(N+1) \times 3(N+1), 3(N+1) \times 1$ and $3(N+1) \times 1$, respectively.

The matrix equation (20) corresponding to the system (9) can be written in the form as

$$
\mathbf{W A}=\mathbf{G} \text { or }[\mathbf{W} ; \mathbf{G}]
$$

where

$$
\mathrm{W}=\mathrm{X} \overline{\mathrm{B}}-\mathrm{PX}=\left[w_{p, q}\right], \quad p, q=1,2, \ldots, 3(N+1)
$$

are the unknown Taylor coefficients. By using the conditions given by (12) and the relations (14), the matrix form for the conditions is obtained as

$$
\overline{\mathrm{X}}(a) \mathrm{A}=\mathrm{C}
$$

where

$$
\mathrm{C}=\left[\begin{array}{lll}
c_{1} & c_{2} & c_{3}
\end{array}\right]^{T}
$$

Hence, the fundamental matrix form for conditions is

$$
\mathbf{U A}=\mathbf{C} \text { or }[\mathbf{U} ; \mathbf{C}]
$$

such that

$$
\mathrm{U}=\overline{\mathrm{X}}(a)
$$

Consequently, we obtain the Taylor polynomial solution of the system (9) under the initial conditions (10) by replacing the row matrices (22) with last rows of the matrix (21). Then, we obtain the new augmented matrix

$$
\widetilde{\mathrm{W}} \mathrm{A}=\widetilde{\mathrm{G}} \text { or }[\widetilde{\mathrm{W}} ; \widetilde{\mathrm{G}}]
$$


If $\operatorname{rank} \widetilde{\mathrm{W}}=\operatorname{rank}[\widetilde{\mathrm{W}} ; \widetilde{\mathrm{G}}]=3(N+1)$, then the unknown Taylor coefficients matrix A is determined as

$$
\mathrm{A}=(\widetilde{\mathrm{W}})^{-1} \widetilde{\mathrm{G}}
$$

By substituting the coefficients $a_{i, 0}, a_{i, 1}, \ldots, a_{i, N}(i=1,2,3)$ into Eq.(11), we can find the Taylor polynomial solutions as

$$
y_{i, N}(x)=\sum_{n=0}^{N} a_{i, N} x^{N}, \quad(i=1,2,3)
$$

\section{Application of Taylor collocation method to determine constant breadth curve pair on a surface}

In this section, we give an example to illustrate the efficiency of the approximation method based on Taylor polynomials used to find approximate solutions of system of differential equations characterizing constant breadth curve mate of a curve on a surface.

Let given a surface of helicoid and a curve on this surface expressed by the following equations

$$
X(u, v)=(u \cos v, u \sin v, v),(-2 \leq u \leq 2,-\pi / 2 \leq v \leq \pi / 2)
$$

and

$$
\alpha(t)=\left(t^{2} \cos t, t^{2} \sin t, t\right),(0 \leq t \leq 2 \pi / 5)
$$

respectively. By using the definition of Darboux frame mentioned in Section 2, we can obtain the elements of Darboux frame of the curve $\alpha(t)$ as

$$
\begin{gathered}
T(t)=\frac{1}{\sqrt{t^{4}+4 t^{2}+1}}\left(2 t \cos t-t^{2} \sin t, 2 t \sin t+t^{2} \cos t, 1\right), \\
g(t)=\frac{1}{\sqrt{t^{4}+1} \sqrt{t^{4}+4 t^{2}+1}}\left(-\cos t-2 t^{3} \sin t-t^{4} \cos t,-t^{4} \sin t+2 t^{3} \cos t-\sin t, 2 t\right), \\
n_{\alpha}(t)=\frac{1}{\sqrt{t^{4}+1}}\left(\sin t,-\cos t, t^{2}\right) .
\end{gathered}
$$

The geodesic curvature, the normal curvature, and the geodesic torsion of the curve $\alpha(t)$ can also be found as

$$
\begin{gathered}
k_{g}=\frac{t^{6}+6 t^{4}+t^{2}-2}{\sqrt{t^{4}+1}\left(t^{4}+4 t^{2}+1\right)^{3 / 2}}, \\
k_{n}=-\frac{4 t}{\sqrt{t^{4}+1}\left(t^{4}+4 t^{2}+1\right)} \\
\tau_{g}=\frac{t^{4}-4 t^{2}+1}{\left(t^{4}+1\right)\left(t^{4}+4 t^{2}+1\right)},
\end{gathered}
$$

respectively. We can express constant breadth curve mate of the curve $\alpha(t)$ as

$$
\alpha^{*}\left(t^{*}\right)=\alpha(t)+\lambda_{1}(t) T(t)+\lambda_{2}(t) g(t)+\lambda_{3}(t) n_{\alpha}(t)
$$


where $\lambda_{i}(t),(i=1,2,3)$ are the unknown functions which should be found. By using the curvatures of the curve $\alpha(t)$ and assuming the condition $\frac{d t^{*}}{d t}=-1$, we obtain the following system of differential equations characterizing the constant breadth curve mate of the curve $\alpha(t)$

$$
\left\{\begin{array}{l}
\lambda_{1}^{\prime}(t)=\frac{t^{6}+6 t^{4}+t^{2}-2}{\sqrt{t^{4}+1}\left(t^{4}+4 t^{2}+1\right)} \lambda_{2}(t)-\frac{4 t}{\sqrt{t^{4}+1} \sqrt{t^{4}+4 t^{2}+1}} \lambda_{3}(t) \\
\lambda_{2}^{\prime}(t)=-\frac{t^{6}+6 t^{4}+t^{2}-2}{\sqrt{t^{4}+1}\left(t^{4}+4 t^{2}+1\right)} \lambda_{1}(t)+\frac{t^{4}-4 t^{2}+1}{\left(t^{4}+1\right) \sqrt{t^{4}+4 t^{2}+1}} \lambda_{3}(t) \\
\lambda_{3}^{\prime}(t)=\frac{4 t}{\sqrt{t^{4}+1} \sqrt{t^{4}+4 t^{2}+1}} \lambda_{1}(t)-\frac{t^{4}-4 t^{2}+1}{\left(t^{4}+1\right) \sqrt{t^{4}+4 t^{2}+1}} \lambda_{2}(t)
\end{array}\right.
$$

The system (23) is a system of differential equations with variable coefficient. It is difficult to find analytical solutions of this system. We can find approximate solutions of the system (23) by using Taylor collocation method described in Section 4 under the initial conditions defined by

$$
\lambda_{1}(0)=2, \lambda_{2}(0)=1, \lambda_{3}(0)=3
$$

These initial conditions have a geometric interpretation such that we can find the distance between the curves $\alpha(t)$ and $\alpha^{*}\left(t^{*}\right)$ at initial point $t=0\left(t^{*}=0\right)$ by using these conditions as

$$
\left\|\alpha^{*}(0)-\alpha(0)\right\|=\sqrt{\lambda_{1}^{2}(0)+\lambda_{2}^{2}(0)+\lambda_{3}^{2}(0)}=c,
$$

where $c=3.741657387$ is the constant distance between the curves $\alpha(t)$ and $\alpha^{*}\left(t^{*}\right)$. By using Taylor collocation method, we obtain $\lambda_{i}(t),(i=1,2,3)$ approximately. Since analytical solutions of the system are not known, we use distance function $d(t)=\left\|\alpha^{*}(t)-\alpha(t)\right\|=\sqrt{\lambda_{1}^{2}(t)+\lambda_{2}^{2}(t)+\lambda_{3}^{2}(t)}$ to control the accuracy of the solutions. Since the curves are constant breadth curve pair, the distance between the corresponding points of the curves should be constant for all $t \in[0,2 \pi / 5]$. Thus, we can find the absolute error of approximate solutions obtained from Taylor collocation method by comparing the constant distance $c$ which is known from initial conditions.

The approximate solutions $\lambda_{1,3}(t), \lambda_{2,3}(t), \lambda_{3,3}(t)$ by the truncated Taylor series for $N=3$ are given by

$$
\lambda_{i, 3}(t)=\sum_{n=0}^{3} a_{i, n} t^{n}, \quad(i=1,2,3) .
$$

The set of the collocation points for $a=0, b=\frac{2 \pi}{5}$ and $N=3$ is calculated as

$$
\left\{t_{0}=0, t_{1}=\frac{2 \pi}{15}, t_{2}=\frac{4 \pi}{15}, t_{3}=\frac{2 \pi}{5}\right\}
$$

By using the Taylor collocation technique described in Section 4, the approximate solutions of the system (23) for $N=3$ are obtained as

$$
\begin{aligned}
& \lambda_{1,3}=2-2 t-8.726196696 t^{2}+6.798258238 t^{3}, \\
& \lambda_{2,3}=1+7 t-10.44465286 t^{2}+4.823723197 t^{3}, \\
& \lambda_{3,3}=3-t+1.583886831 t^{2}-1.439830030 t^{3} .
\end{aligned}
$$


The comparison of the distance function obtained from approximate solutions for $N=3$ and the constant breadth $c$ can be seen in Table 1.

The set of the collocation points for $a=0, b=\frac{2 \pi}{5}$ and $N=4$ is calculated as

$$
\left\{t_{0}=0, t_{1}=\frac{\pi}{10}, t_{2}=\frac{\pi}{5}, t_{3}=\frac{3 \pi}{10} t_{4}=\frac{2 \pi}{5}\right\}
$$

By using the Taylor collocation technique, the approximate solutions of the system (23) for $N=4$ are obtained as

$$
\begin{aligned}
& \lambda_{1,4}=2-2 t-13.99013604 t^{2}+18.92678564 t^{3}-6.932188065 t^{4}, \\
& \lambda_{2,4}=1+7 t-8.425862894 t^{2}+0.204720105 t^{3}+2.573201945 t^{4}, \\
& \lambda_{3,4}=3-t+1.471888354 t^{2}-2.546105833 t^{3}+1.085891320 t^{4} .
\end{aligned}
$$

The comparison of the distance function obtained from approximate solutions for $N=4$ and the constant breadth $c$ can be seen in Table 2.

The set of the collocation points for $a=0, b=\frac{2 \pi}{5}$ and $N=5$ is calculated as

$$
\left\{t_{0}=0, t_{1}=\frac{2 \pi}{25}, t_{2}=\frac{4 \pi}{25}, t_{3}=\frac{6 \pi}{25}, t_{4}=\frac{8 \pi}{25}, t_{5}=\frac{2 \pi}{5}\right\}
$$

By using the Taylor collocation technique, the approximate solutions of the system (23) for $N=5$ are obtained as

$$
\begin{aligned}
& \lambda_{1,5}=2-2 t-16.01444043 t^{2}+26.29588723 t^{3}-16.10226871 t^{4}+3.807538795 t^{5}, \\
& \lambda_{2,5}=1+7 t-4.183603447 t^{2}-16.40261814 t^{3}+24.02114642 t^{4}-8.973032078 t^{5} \\
& \lambda_{3,5}=3-t+0.530344699 t^{2}+0.1901936784 t^{3}-1.897904809 t^{4}+1.127048776 t^{5} .
\end{aligned}
$$

The comparison of the distance function obtained from approximate solutions for $N=5$ and the constant breadth $c$ can be seen in Table 3.

The set of the collocation points for $a=0, b=\frac{2 \pi}{5}$ and $N=6$ is calculated as

$$
\left\{t_{0}=0, t_{1}=\frac{\pi}{15}, t_{2}=\frac{2 \pi}{15}, t_{3}=\frac{\pi}{5}, t_{4}=\frac{4 \pi}{15},, t_{5}=\frac{\pi}{3} t_{6}=\frac{2 \pi}{5}\right\}
$$

By using the Taylor collocation technique, the approximate solutions of the system (23) for $N=6$ are obtained as

$$
\begin{aligned}
& \lambda_{1,6}=2-2 t-15.42148709 t^{2}+23.06443038 t^{3}-9.799241814 t^{4}-1.431077548 t^{5}+1.576173710 t^{6}, \\
& \lambda_{2,6}=1+7 t-2.089908983 t^{2}-27.67621133 t^{3}+47.10766701 t^{4}-29.63709927 t^{5}+6.762066403 t^{6}, \\
& \lambda_{3,6}=3-t+0.159351405 t^{2}+2.181206439 t^{3}-6.131673130 t^{4}+5.114820336 t^{5}-1.371285620 t^{6} .
\end{aligned}
$$

The comparison of the distance function obtained from approximate solutions for $N=6$ and the constant breadth $c$ can be seen in Table 4. 
Table 1: The absolute error of the approximate solutions of the Taylor collocations method for $N=3$.

\begin{tabular}{|c|c|c|c|c|c|}
\hline$t_{i}$ & $\lambda_{1,3}\left(t_{i}\right)$ & $\lambda_{2,3}\left(t_{i}\right)$ & $\lambda_{3,3}\left(t_{i}\right)$ & $d_{i}$ & $\left|d_{i}-c\right|$ \\
\hline 0 & 2 & 1 & 3 & 3.741657387 & 0 \\
$\pi / 25$ & 1.624364488 & 1.724282801 & 2.896490831 & 3.741840496 & 0.000183109 \\
$2 \pi / 25$ & 1.054074677 & 2.176128134 & 2.825861932 & 3.719153498 & 0.022503889 \\
$3 \pi / 25$ & 0.3700734212 & 2.412969229 & 2.770970095 & 3.692918913 & 0.048738474 \\
$4 \pi / 25$ & -0.3466964314 & 2.49223931 & 2.714672113 & 3.701472664 & 0.040184723 \\
$\pi / 5$ & -1.015292028 & 2.471371610 & 2.639824781 & 3.755951332 & 0.014293945 \\
$6 \pi / 25$ & -1.554770516 & 2.407799351 & 2.529284889 & 3.822576503 & 0.080919116 \\
$7 \pi / 25$ & -1.884189046 & 2.358955761 & 2.365909233 & 3.835670364 & 0.094012977 \\
$8 \pi / 25$ & -1.922604764 & 2.382274065 & 2.132554604 & 3.730874956 & 0.010782431 \\
$9 \pi / 25$ & -1.589074814 & 2.535187497 & 1.812077794 & 3.497993759 & 0.243663628 \\
$2 \pi / 5$ & -0.80265635 & 2.875129283 & 1.387335598 & 3.291705587 & 0.449951800 \\
\hline
\end{tabular}

Table 2: The absolute error of the approximate solutions of the Taylor collocations method for $N=4$.

\begin{tabular}{|c|c|c|c|c|c|}
\hline$t_{i}$ & $\lambda_{1,4}\left(t_{i}\right)$ & $\lambda_{2,4}\left(t_{i}\right)$ & $\lambda_{3,4}\left(t_{i}\right)$ & $d_{i}$ & $\left|d_{i}-c\right|$ \\
\hline 0 & 2 & 1 & 3 & 3.741657387 & 0 \\
$\pi / 25$ & 1.563578900 & 1.747637968 & 2.892797712 & 3.723881852 & 0.017775535 \\
$2 \pi / 25$ & 0.8864598746 & 2.240585044 & 2.805557703 & 3.698268037 & 0.043389350 \\
$3 \pi / 25$ & 0.1317612114 & 2.504378914 & 2.717713278 & 3.698004897 & 0.043652490 \\
$4 \pi / 25$ & -0.5788866667 & 2.579957399 & 2.615196604 & 3.718943833 & 0.022713554 \\
$\pi / 5$ & -1.165341195 & 2.523658461 & 2.490438703 & 3.732178594 & 0.009478793 \\
$6 \pi / 25$ & -1.588947669 & 2.407220193 & 2.342369457 & 3.715663929 & 0.025993458 \\
$7 \pi / 25$ & -1.852539254 & 2.317780827 & 2.176417605 & 3.679783043 & 0.061874344 \\
$8 \pi / 25$ & -2.000436929 & 2.357878733 & 2.004510742 & 3.685024198 & 0.056633189 \\
$9 \pi / 25$ & -2.11844963 & 2.645452421 & 1.845075328 & 3.858827584 & 0.117170197 \\
$2 \pi / 5$ & -2.33387403 & 3.313840520 & 1.723036673 & 4.404243676 & 0.662586289 \\
\hline
\end{tabular}

Table 3: The absolute error of the approximate solutions of the Taylor collocations method for $N=5$.

\begin{tabular}{|c|c|c|c|c|c|}
\hline$t_{i}$ & $\lambda_{1,5}\left(t_{i}\right)$ & $\lambda_{2,5}\left(t_{i}\right)$ & $\lambda_{3,5}\left(t_{i}\right)$ & $d_{i}$ & $\left|d_{i}-c\right|$ \\
\hline 0 & 2 & 1 & 3 & 3.741657387 & 0 \\
$\pi / 25$ & 1.544068221 & 1.786740647 & 2.882650626 & 3.726427705 & 0.015229682 \\
$2 \pi / 25$ & 0.8428103816 & 2.321481107 & 2.778749183 & 3.717667399 & 0.023989988 \\
$3 \pi / 25$ & 0.08265966223 & 2.582390840 & 2.678819941 & 3.721780669 & 0.019876718 \\
$4 \pi / 25$ & -0.6176843455 & 2.623917574 & 2.570504786 & 3.724778146 & 0.016879241 \\
$\pi / 5$ & -1.192937885 & 2.543045384 & 2.442801371 & 3.722560834 & 0.019096553 \\
$6 \pi / 25$ & -1.616915264 & 2.445552771 & 2.290301211 & 3.720298773 & 0.021358614 \\
$7 \pi / 25$ & -1.888211119 & 2.412270753 & 2.117427821 & 3.723962867 & 0.017695520 \\
$8 \pi / 25$ & -2.015882626 & 2.465340916 & 1.942674825 & 3.730371841 & 0.011285546 \\
$9 \pi / 25$ & -2.005131834 & 2.53447355 & 1.802844083 & 3.700588688 & 0.041068699 \\
$2 \pi / 5$ & -1.84298780 & 2.42320566 & 1.757283811 & 3.515192184 & 0.226465203 \\
\hline
\end{tabular}


Table 4: The absolute error of the approximate solutions of the Taylor collocations method for $N=6$.

\begin{tabular}{|c|c|c|c|c|c|}
\hline$t_{i}$ & $\lambda_{1,6}\left(t_{i}\right)$ & $\lambda_{2,6}\left(t_{i}\right)$ & $\lambda_{3,6}\left(t_{i}\right)$ & $d_{i}$ & $\left|d_{i}-c\right|$ \\
\hline 0 & 2 & 1 & 3 & 3.741657387 & 0 \\
$\pi / 25$ & 1.548433072 & 1.802567720 & 2.879806902 & 3.733655469 & 0.008001918 \\
$2 \pi / 25$ & 0.8492569158 & 2.347854943 & 2.773683963 & 3.731887307 & 0.009770080 \\
$3 \pi / 25$ & 0.08574104898 & 2.604304372 & 2.673682201 & 3.733407197 & 0.008250190 \\
$4 \pi / 25$ & -0.6185719159 & 2.640939138 & 2.565199060 & 3.733285566 & 0.008371821 \\
$\pi / 5$ & -1.194075520 & 2.563831749 & 2.436492195 & 3.733034128 & 0.008623259 \\
$6 \pi / 25$ & -1.614839862 & 2.471790208 & 2.284305276 & 3.733028959 & 0.008628428 \\
$7 \pi / 25$ & -1.883883169 & 2.431156258 & 2.115605892 & 3.733004800 & 0.008652587 \\
$8 \pi / 25$ & -2.013974474 & 2.469820915 & 1.945435479 & 3.733741787 & 0.007915600 \\
$9 \pi / 25$ & -2.003967523 & 2.59039395 & 1.790871331 & 3.732726453 & 0.008930934 \\
$2 \pi / 5$ & -1.810665726 & 2.80254874 & 1.661100674 & 3.727203410 & 0.014453977 \\
\hline
\end{tabular}

The comparison of the distance function, which consist of approximate solutions obtained by Taylor collocation method for $N=3,4,5,6$, and the constant breadth $c$, which is found by initial conditions, can be seen in Figure 1.

It is seen from Figure 1 that accuracy of the solution of system (23) increase when the value of $N$ is increased. Thus, the distance function $d(t)=\sqrt{\lambda_{1}^{2}(t)+\lambda_{2}^{2}(t)+\lambda_{3}^{2}(t)}$ obtained by Taylor collocation method gets closer to the constant breadth $c$. Therefore, it can be said that this method is effective to find approximate solutions of system (23) which is used to find a constant breadth curve mate of the curve on the surface.

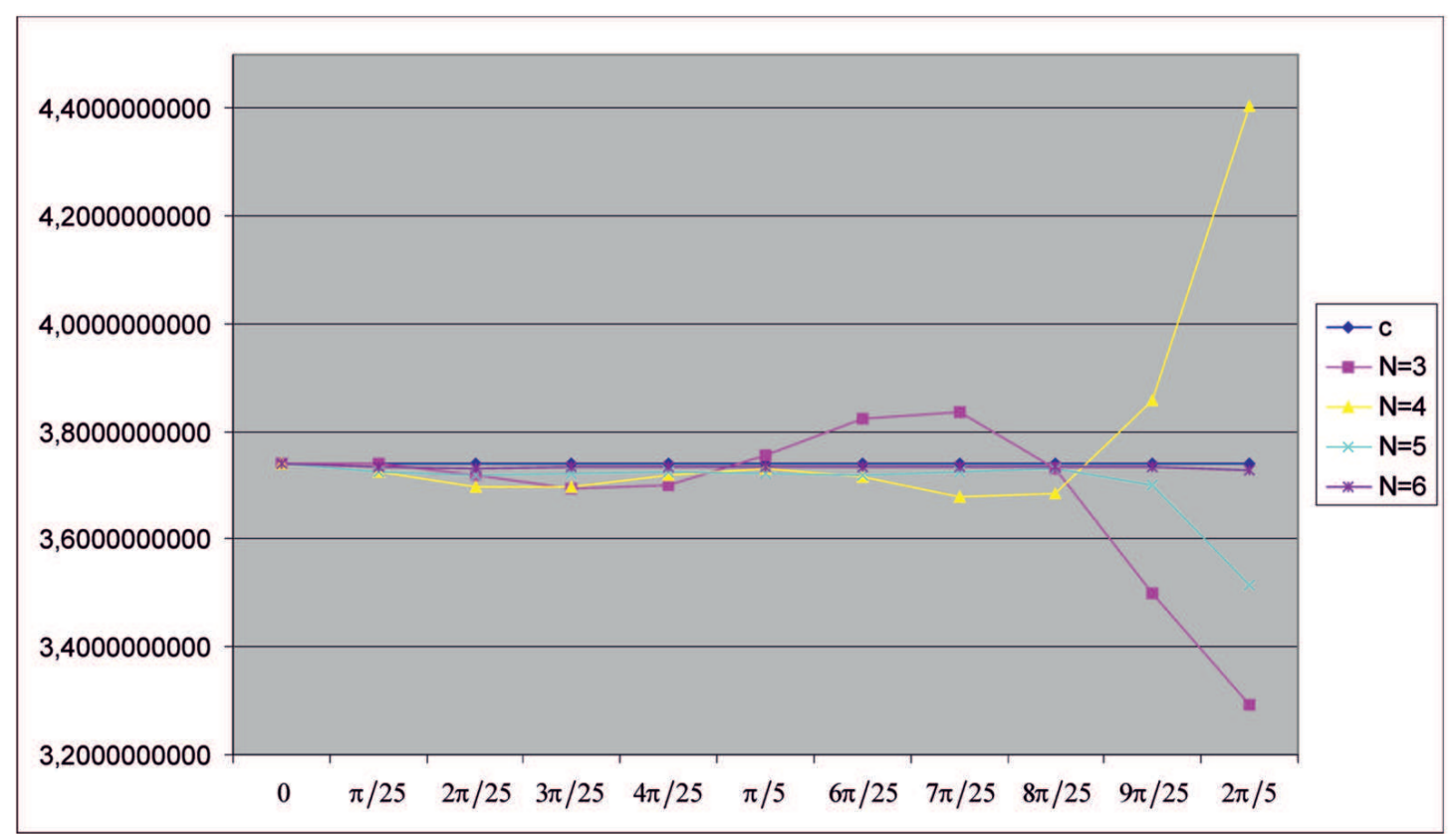

Fig. 1: Comparison of the approximate solutions for $N=3,4,5,6$.

Now, we can approximately draw the constant breadth curve mate $\alpha^{*}$ of the curve $\alpha$ on the surface of helicoid by using the Taylor collocations method for $N=6$ (see Figure 2). 


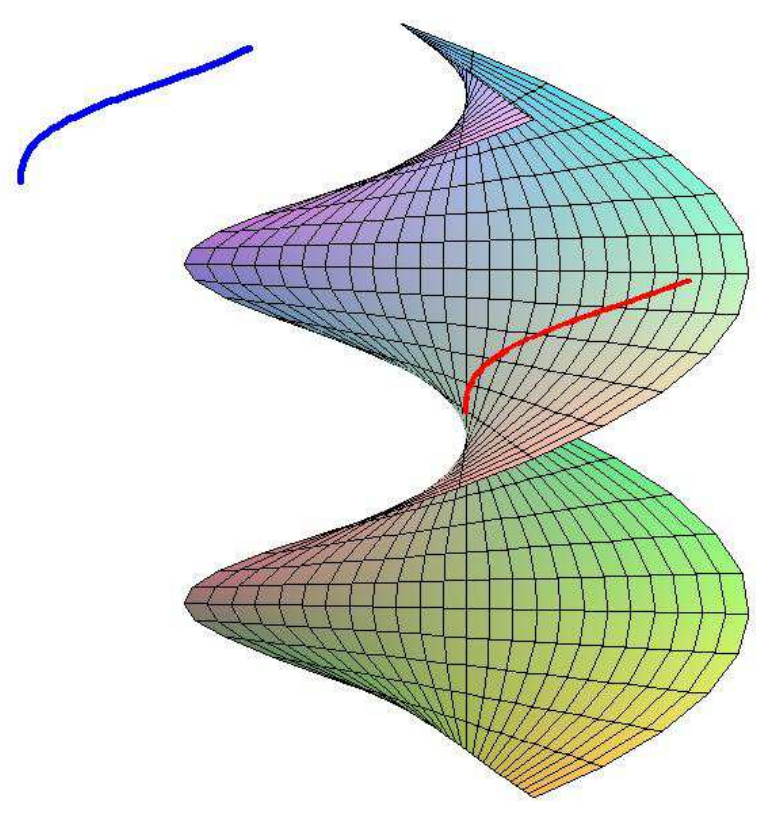

Fig. 2: The curve $\alpha$ (red curve) on the surface of helicoid and its constant breadth curve mate $\alpha^{*}$ (blue curve) for $N=6$.

\section{Conclusions}

In this paper, we give a system of differential equations characterizing constant breadth curve mate of a curve on a surface. This system of differential equations has variable coefficients. It is usually difficult to find analytical solutions of the system. So, we use Taylor collocation method to obtain approximate solutions of the system. We give an example to show the efficiency of the method. The approximate solutions are obtained, and the graphics are drawn easily by using the computer program Maple12. It is seen from Tables 1-4 and Figure 1 that the distance function obtained from approximate solutions are very close to constant breadth when the values of $N$ are selected big enough. In other words, it can be said that the errors decrease when the values of $N$ increase. This method can also be used to find approximate solutions of other problems similar to our problem. But some modifications are required.

\section{Competing interests}

The authors declare that they have no competing interests.

\section{Authors' contributions}

All authors have contributed to all parts of the article. All authors read and approved the final manuscript.

\section{References}

[1] Akdoğan, Z., Mağden, A., Some characterization of curves of constant breadth in $E^{n}$ space, Turk J Math, 25: 433-444, 2001. 
[2] Barbier, E., Note sur le probléme de I?aiguille et le jeu du joint couvert, Journal de mathématiques pures et appliquées, 2(5): 273-286, 1860.

[3] Blaschke, W., Leibziger Berichte, 67: 290, 1917.

[4] Çetin, M., Kocayiğit, H., Sezer, M., On the solution of differential equation system characterizing curve pair of constant breadth by the Lucas collocation approximation, New Trends in Mathematical Sciences, 4(1): 168-183, 2016.

[5] Çetin, M., Sezer, M., Kocayiğit, H., Determination of the curves of constant breadth according to Bishop Frame in Euclidean 3-space, New Trends in Mathematical Sciences, 3(3): 18-34, 2015.

[6] Euler, L., De curvis triangularibus, Acta Acad. Petropol., 2: 3-30, 1778, 1780.

[7] Fujivara, M., On space curves of constant breadth, Tohoku Math. J., 5: 179-184, 1914.

[8] Kazaz, M., Önder, M., Kocayiğit, H., Spacelike curves of constant breadth in Minkowski 4-space, Int. J. Math. Anal, 2(22): 10611068, 2008.

[9] Kocayiğit, H., Çetin, M., Space curves of constant breadth according to Bishop frame in Euclidean 3-space, New Trends in Mathematical Sciences, 2(3): 199-205, 2014.

[10] Kocayiğit, H., Çiçek, Z., Some characterizations of constant breadth spacelike curves in Minkowski 4-space, New Trends in Mathematical Sciences, 3(2): 1-12, 2015.

[11] Kocayiğit, H., Önder, M., Space curves of constant breadth in Minkowski 3-space, Annali di Matematica Pura ed Applicata, 192(5): 805-814, 2013.

[12] Köse, Ö., On space curves of constant breadth, Doğa Tr. J. Math, 10(1): 11-14, 1986.

[13] Köse, Ö., Some properties of ovals and curves of constant width in a plane, Doğa Mat., (8)2: 119-126, 1984.

[14] Mağden, A., Köse, Ö., On the curves of constant breadth in $E^{4}$ space, Turkish Journal of Mathematics, 21(3): 277-284, 1997.

[15] Mellish, A.P., Notes on differential geometry, Annals of Mathematics, 32(1): 181-190, 1931.

[16] Önder, M., Kocayiğit, H., Candan, E., Differential equations characterizing timelike and spacelike curves of constant breadth in Minkowski 3-Space E ${ }_{1}^{3}$, J. Korean Math. Soc., 48(4): 849-866, 2011.

[17] Reuleaux, F., The kinematics of machinery, Trans. by A. B. W. Kennedy, Dover Pub., New York, 1963.

[18] Sezer, M., Differential equations characterizing space curves of constant breadth and a criterion for these curves, Turkish J. of Math, 13(2): 70-78, 1989.

[19] Sezer, M., Karamete, A., Gülsu, M., Taylor polynomial solutions of systems of linear differential equations with variable coefficients, International Journal of Computer Mathematics, 82(6): 755-764, 2005.

[20] Y1lmaz, S., Turgut, M., Partially null curves of constant breadth in semi-Riemannian space, Modern Applied Science, 3(3): 60-63, 2009. 